\title{
ASSOCIATION BETWEEN PHYSICAL MULTIMORBIDITY AND SUICIDAL IDEATION IN YOUNG ADULTS WITH OBESITY
}

Joanne Mattar ${ }^{1,2}$, Valérie Chauvin ${ }^{1,2}$, Jacques Marleau $^{3}$, Katerina Kavalidou ${ }^{4}$, A.J. Romain ${ }^{1,2}$.

${ }^{1}$ School of Kinesiology and Physical Activity Sciences, University of Montreal, Montreal, Canada.

${ }^{2}$ Centre de recherche de l'Institut universitaire en santé mentale de Montréal, Montréal, Canada

${ }^{3}$ Centre intégré de santé et de services sociaux de l'Outaouais, Gatineau, Canada.

${ }^{4}$ National Suicide Research Foundation, World Health Organisation (WHO) Collaborating Center for Surveillance and Research in Suicide Prevention, University College Cork, Ireland

Corresponding author:

Ahmed Jerome ROMAIN, PhD

School of kinesiology and physical activity sciences

University of Montreal

2100 Boulevard Edouard Montpetit,

Montreal, H3T 1J4, Qc Canada

Montreal Mental Health Institute Research Center

aj.romain@umontreal.ca

Please cite as Mattar, J., Chauvin, V., Marleau, J., Kavalidou, K., \& Romain, A. J. (2021). Association between physical multimorbidity and suicidal ideation in young adults with obesity. 


\section{ASSOCIATION BETWEEN PHYSICAL MULTIMORBIDITY AND SUICIDAL IDEATION IN YOUNG ADULTS WITH OBESITY}

\section{Abstract \\ Background}

Obesity is considered as one of the entrance point of multimorbidity and has an impact on physical and mental health. While some evidence points out to a possible relationship between obesity, multimorbidity and suicidal spectrum, little provide a direct association. Thus, the aim of the present study was to examine the co-occurring effect of both multimorbidity and obesity on suicidal ideation.

\section{Methods}

A cross-sectional analysis of the Canadian Community Health Survey data was conducted. A weighted sample of young adults (18 to 30 years-old) with obesity, from the province of Quebec, of the $2005 \quad(n=394)$ and 2015-2016 ( $\mathrm{n}=295)$ cycles were investigated independently. Multimorbidity, suicidal ideation, and health behaviours were self-reported.

\section{Results}

The prevalence of physical multimorbidity was $15 \%$ in 2005 and $18 \%$ in $2015-2016$. Adjusted logistic regressions showed an association between multimorbidity and suicidal ideation (2005: OR 3.59, 95\% CI 1.89-6.81; 2015-2016: OR 3.72, 95\% CI 1.88-7.36). Among covariates, the significant association of sex (OR 1.98; 95\% CI 1.16-3.37) and educational status (OR 3.27; 95\% CI 1.49-7.18) in the 2005 cycle, were not replicated in the 2015-2016 cycle (education: OR 0.93; 95\% CI 0.46-1.87; sex (OR 0.90; 95\% CI 0.481.69). Finally, our results suggest no consistent association between health behaviours and suicidal ideation.

\section{Conclusion}

Multimorbidity seems to be associated with suicidal ideation among those with obesity. Attention should be given to multimorbidity management within obesity-related 
interventions for young people, as the development of suicidal ideation may also be prevented.

Keywords

Suicidal behaviour; Multimorbidity; Obesity; Young adults. 


\section{ASSOCIATION BETWEEN PHYSICAL MULTIMORBIDITY AND SUICIDAL IDEATION IN YOUNG ADULTS WITH OBESITY}

\section{INTRODUCTION}

Described by the World Health Association (WHO) as a "global phenomenon"(WHO, 2021), suicide is one of the leading causes of death internationally, particularly among young and middle-aged adults (Arensman et al., 2020). Suicidal behaviour goes beyond the act of taking one's life, and encompasses the thoughts, drive, ideation, and attempts behind the act itself, as well as self-harming (Pediatric Clinical Advisor, 2007).

As the ninth cause of mortality among Canadians, an approximate average of 10 people dies each day by suicide as stated by the Public Health Agency of Canada (Canada, 2020). For Canadian youth, aged 15 to 24 years, suicide is the second leading cause of death, as this delicate transitional phase implies numerous biological, social, and psychological challenges are faced.

Numerous biopsychosocial factors are associated with suicidal behaviours (Kavalidou et al., 2019; Vilhjalmsson et al., 1998), some of which are adverse life events (e.g. total life, financial, family or legal stress, etc.), poor social support (e.g. emotional, informational or material support), personality traits (e.g. self-esteem, external locus of control), distress (e.g. fatigue, hopelessness, loneliness, depression, anxiety), chronic physical diseases (e.g. asthma, arthritis, diabetes, cancer) as well as mental diseases (including schizophrenia spectrum disorder, and mood disorders) (Kavalidou et al., 2019; Vilhjalmsson et al., 1998). Furthermore, the role of health behaviours in worsening the impact of the previous listed factors, include low physical activity levels, excessive smoking, poor diet, and substance use (Berardelli et al., 2018). Other socio-demographic factors may also affect one's suicidal risk such as gender, age, education level, annual individual income, marital status, and employment (Vilhjalmsson et al., 1998). 
Among the different health conditions, obesity, defined as an "abnormal or excessive fat accumulation (Body Mass Index, BMI $\geq 30 \mathrm{~kg} / \mathrm{m}^{2}$ ) presenting a risk to health" (Fruh, 2017; WHO, 2016) has been shown to be associated with suicidal behaviour (Perera et al., 2016). However, the true nature of this association remains controversial given its different influence on distinct phases of the suicidality spectrum (Perera et al., 2016). In fact, the social stigma, psychological distress, and physical comorbidities associated with obesity increase the susceptibility of suicidal ideation (Romain et al., 2018). Furthermore, the relationship between BMI and suicide attempt is dependent on multiple confounding factors such as sex, age, multimorbidity and body weight (Perera et al., 2015).

As indicated in several studies, multimorbidity, defined as the co-occurrence of two and more chronic diseases (Navickas et al., 2016), is one risk factor known to have an association with suicidal ideation (Kavalidou et al., 2019, 2016), with both physical and mental health conditions found to be further associated with deaths by suicide (Qin et al., 2014; Rockett et al., 2007). Previous research has identified 63 physical (including obesity) and 36 mental illnesses associated with suicide(Harris and Barraclough, 1997, 1994).

However, despite the obvious knowledge of an association between obesity, multimorbidity and suicidal behaviours, few studies explored the co-occurrence of these factors simultaneously. Whereof, the aim of the present study was to understand the association between multimorbidity and suicidal ideation among young adults with obesity.

\section{METHODS}

\section{Procedure and settings}

The current study was based on data from the Canadian Community Health Survey (CCHS) conducted in 2005 and 2015-2016. This health population survey provides cross-sectional information related to health status, health care utilization and health determinants in the Canadian population. The CCHS sample is an administrative database representing $98 \%$ 
of the Canadian population aged 12 years or older living in private dwellings in ten provinces and three territories, excluding Indian Reserves or Crown lands, residents in institutions and certain remote regions, as well as full-time members of the Canadian Forces. Respondents were interviewed by telephone or face-to-face. All data were selfreported.

\section{Study-specific inclusion criteria}

In the present study, our sample consisted of young adults (ages 18 to 30 years old) with obesity $\left(\mathrm{BMI} \geq 30 \mathrm{~kg} / \mathrm{m}^{2}\right)$, inhabitants from the province of Quebec who provided complete answers to the questionnaires.

BMI was calculated (weight $(\mathrm{kg}) /$ height $\left(\mathrm{m}^{2}\right)$ ) based on self-reported weight and height and was divided into three classes being class I (30.0-34.9 kg/m²), class $\Pi$ (35.0 to 39.9) and class $\amalg\left(\geq 40.0 \mathrm{~kg} / \mathrm{m}^{2}\right)$.

\section{Measurements}

\section{a) Suicidal ideation}

Participants were questioned on their suicidal ideation and suicide attempts during the past year, for which they may or may not have consulted health professionals. The items were formulated as follows, "Have you ever seriously considered committing/ attempted to commit suicide or taking your own life? Has this happened in the past 12 months?". In the present study, this past year question on suicidal ideation and attempts was used.

\section{b) Physical comorbidities and multimorbidity definition}

Participants were asked about any physical health condition. Self-reported data concerning health conditions were diagnosed by a health professional and expected to last or have lasted 6 months or more. In the present study, 11 different physical conditions were selected: asthma, arthritis, type 2 diabetes, hypertension, migraines, heart diseases, cancer, digestive disorders, stomach ulcers, cerebrovascular disorders, and back aches. 
Multimorbidity was defined by the presence of two or more physical conditions except for obesity, which was the main health condition investigated.

\section{c) Health behaviours}

To evaluate the potential moderating impact of health behaviours on the association between obesity, multimorbidity, and suicidal ideation, physical activity (PA), fruits and vegetables consumption, and tobacco was evaluated.

PA, defined as leisure-time activities, was evaluated based on its nature, duration, and frequency during the past three months. Then, following the index of the metabolic equivalent of each PA, three categories were identified in the 2005 cycle: inactive $(<1.5 \mathrm{kcal} / \mathrm{kg} / \mathrm{d})$, moderately active ( 1.5 to $<3 \mathrm{kcal} / \mathrm{kg} / \mathrm{d})$, and active ( $\geq 3 \mathrm{kcal} / \mathrm{kg} / \mathrm{d})$. In the 2015-2016 CCHS cycle, leisure-time PA was also evaluated.

Fruits and vegetables consumption were evaluated based on the portion size per day. Two groups were identified whether they meet the Canadian food guidelines at the time of evaluation.

Reported smoking habits of the study participants led to their division in three groups: regular smokers (daily), occasional smokers (not daily or less than 100 cigarettes in a lifetime) and non-smokers.

\section{d) Covariates}

The covariates in this study included the demographics of sex, age and education.

\section{Statistical analyses}

In the present study, we used data from the province of Quebec. To ensure a representative sample, three samples' grounds were used to select the sample of the following households (49\% using an areolar frame, 50\% using a list frame of telephone numbers and $1 \%$ using a random digit dialing). Moreover, according to the CCHS, each province is divided into three regions: major urban centers, cities and rural regions including different geographical and socioeconomic strata. Thus, to better represent the Quebec population, the weight 
given to each participant has been normalized through the division of the raw number by its mean and later by the overall effect.

To understand the influence of obesity and multimorbidity on suicidal ideation, two models of logistic regressions were run. In the first model, we examined the effects of the independent variables (BMI classes and multimorbidity) on the dependent variable (suicidal ideation), after adjusting the covariates (age, sex, and education). In the second model, we used the same setting as in the first model, and health behaviours (PA and tobacco consumption only) were added to the previous adjustments. Note that nutrition was not taken into consideration as dietary habits, as it was not evaluated in Quebec in the 2005 cycle.

To overcome the cross-sectional issue, in the present study, an association was considered reliable if it has been duplicated twice in both the 2005 and 2015-2016 cycles. Statistical analyses were performed with SPSS V.21.

\section{RESULTS}

\section{Sample characteristics}

In the 2005 and 2015-2016 CCHS cycles, respectively, 394 and 295 participants with obesity were sampled. Majority of the population was male (2005: 53.0\%; 2015-2016: 48.8\%), non-students (2005: 74.1\%; 2015-2016: 73.2\%), aged between 25 and 29 years old (2005: 53.8\%; 2015-2016: 59.7\%).

In addition, $70.1 \%$ of the 2005 cycle and $67.5 \%$ of the $2015-2016$ cycle suffered from class I obesity $\left(30.0-34.9 \mathrm{~kg} / \mathrm{m}^{2}\right)$ while $29.9 \%$ and $32.5 \%$ of the 2005 and $2015-2016$ samples, respectively, were diagnosed with classes $\Pi\left(35.0\right.$ to $\left.39.9 \mathrm{~kg} / \mathrm{m}^{2}\right)$ and class $\amalg(\geq 40.0$ $\mathrm{kg} / \mathrm{m}^{2}$ ) (Table 1). 
2015-2016

(N=394)

$(\mathrm{N}=\mathbf{2 9 5})$

$\mathbf{N}$

$\%$

N \%

Sex

\begin{tabular}{|c|c|c|c|c|}
\hline Male & 209 & 53.0 & 144 & 48.8 \\
\hline Female & 185 & 47.0 & 151 & 51.2 \\
\hline \multicolumn{5}{|l|}{ Age (years old) } \\
\hline $18-24$ & 182 & 46.2 & 119 & 40.3 \\
\hline $25-29$ & 212 & 53.8 & 176 & 59.7 \\
\hline \multicolumn{5}{|l|}{ Education } \\
\hline Student & 90 & 22.8 & 77 & 26.1 \\
\hline Non-student & 292 & 74.1 & 216 & 73.2 \\
\hline \multicolumn{5}{|l|}{ Multimorbidity } \\
\hline 0-1 Chronic disease & 333 & 84.5 & 240 & 81.4 \\
\hline 2+ Chronic diseases & 59 & 15.0 & 53 & 18.0 \\
\hline \multicolumn{5}{|l|}{ BMI } \\
\hline Class I $\left(30.0-34.9 \mathrm{~kg} / \mathrm{m}^{2}\right)$ & 276 & 70.1 & 199 & 67.5 \\
\hline $\begin{array}{l}\text { Class II }\left(35.0 \text { to } 39.9 \mathrm{~kg} / \mathrm{m}^{2}\right) \text { and } \\
\text { III }\left(\geq 40.0 \mathrm{~kg} / \mathrm{m}^{2}\right)\end{array}$ & 118 & 29.9 & 96 & 32.5 \\
\hline
\end{tabular}

*CCHS: Canadian Community Health Survey; BMI: Body Mass Index

Table 1. Descriptive characteristics of the included sample.

\section{Prevalence of physical multimorbidity}

The prevalence of physical multimorbidity was $15 \%$ in the 2005 cycle, and $18 \%$ in the 2015-2016 cycle. A slight increase in the population's obesity rank was reported with the prevalence of participants with classes $\Pi$ and $\amalg$ was $29.9 \%$ in 2005 and $32.5 \%$ in 20152016. 


\section{Association between suicidal ideation and multimorbidity}

Results showed that participants with obesity and physical multimorbidity were three times more likely to have suicidal ideation (2005: OR 3.59, 95\%CI 1.89-6.81) and this association was confirmed in the 2015-2016 cycle (OR 3.72, 95\%CI 1.88-7.36). Regarding the impact of obesity severity, no associations were found between obesity classes and suicidal ideation across cycles (2005: OR 1.68, 95\%CI 0.98-2.87; 2015-2016: OR 1.31, 95\% CI 0.71-2.42) (Table 2).

\begin{tabular}{lcccccc}
\hline & \multicolumn{2}{c}{$\mathbf{2 0 0 5}$} & & & $\mathbf{2 0 1 5 - 2 0 1 6}$ & \\
& OR & $\mathbf{9 5 \%} \mathbf{C I}$ & P value & OR & $\mathbf{9 5 \%} \mathbf{C I}$ & P value \\
\hline Model 1 & & & & & & \\
\hline Sex & 1.98 & $1.16-3.37$ & 0.01 & 0.90 & $0.48-1.68$ & 0.74 \\
\hline Age & 0.79 & $0.46-1.37$ & 0.41 & 0.96 & $0.51-1.82$ & 0.90 \\
\hline Education & 3.27 & $1.47-7.18$ & 0.003 & 0.93 & $0.46-1.87$ & 0.83 \\
\hline Multimorbidity & 3.59 & $1.89-6.81$ & 0.0001 & 3.72 & $1.88-7.36$ & 0.0001 \\
\hline BMI & 1.68 & $0.98-2.87$ & 0.06 & 1.31 & $0.71-2.42$ & 0.38
\end{tabular}

* OR [95\% CI]: Odds ratio and its 95\% confidence interval; BMI: Body Mass Index

Table 2. Model 1: Association Between Suicidal Ideation and Multimorbidity among Persons with Obesity (BMI $\geq 30 \mathrm{~kg} / \mathrm{m}^{2}$ ) with Adjustment for the covariates. 


\section{Impact of health behaviours on the association between suicidal ideation and multimorbidity}

When the logistic regressions were adjusted for PA and tobacco consumption, young adults with obesity and physical multimorbidity were still more likely to have suicidal ideation (2005: 3.56 95\%CI 1.84-6.88) and this association was also detected in the 2015-2016 cycle (OR: 3.21 95\%CI 1.44-7.12). Regarding health behaviour, only tobacco consumption was associated with suicidal ideation once in the 2015-2016 cycle (20152016: 3.54 95\%CI 1.80-6.99) (Table 3).

\begin{tabular}{rrrrrr}
\hline & & & 2005 \\
OR & $95 \%$ CI & P value & OR & $95 \%$ CI & P value
\end{tabular}

\section{Model 2}

\begin{tabular}{lcccccc}
\hline Sex & 1.97 & $1.15-3.37$ & 0.01 & 0.76 & $0.38-1.53$ & 0.44 \\
& & & & & & \\
\hline Age & 0.75 & $0.43-1.30$ & 0.41 & 1.31 & $0.64-2.70$ & 0.46 \\
& & & & & & \\
\hline Education & 3.25 & $1.48-7.15$ & 0.003 & 0.84 & $0.38-1.88$ & 0.68 \\
& & & & & & \\
\hline Multimorbidity & 3.56 & $1.84-6.88$ & 0.0001 & 3.21 & $1.44-7.12$ & 0.004 \\
& 1.62 & $0.94-2.81$ & 0.06 & 1.51 & $0.77-2.98$ & 0.23 \\
BMI & 1.15 & $0.65-2.01$ & 0.63 & 3.54 & $1.80-6.99$ & 0.0001 \\
\hline Tobacco & 1.59 & $0.92-2.74$ & 0.10 & 1.43 & $0.69-2.95$ & 0.34
\end{tabular}

* OR [95\% CI]: Odds ratio and its 95\% confidence interval; BMI: Body Mass Index; PA: Physical Activity

Table 3. Model 2: Association Between Suicidal Ideation and Multimorbidity among Persons with Obesity (BMI $\geq 30 \mathrm{~kg} / \mathrm{m}^{2}$ ) with Adjustment for the covariates and Health Behaviours. 


\section{DISCUSSION}

The aim of the present study was to investigate the association between multimorbidity and obesity classes with suicidal ideation among young adults with obesity, and whether this association could be replicated. Moreover, we wanted to investigate whether health behaviours could attenuate the association between multimorbidity and suicidal ideation. Briefly, our study provides further evidence on the association between multimorbidity and suicidal ideation (Kavalidou et al., 2019, 2016), particularly, in a young population with obesity (Agborsangaya et al., 2013; Perera et al., 2016, 2015). However, our study contradicts other findings stating the importance of healthy lifestyle in the prevention of suicide, given the lack of association between health behaviours and suicidal ideation (Berardelli et al., 2018).

To start, given the increase in obesity in the Canadian population, the prevalence of physical multimorbidity was higher through the years (15\% in 2005 and $18 \%$ in the 20152016). This finding is not a surprise and further confirms the well-known role of obesity as a predictor of multimorbidity (Agborsangaya et al., 2013; Sailer, 1998) even in young adults. Consequently, our study shows that even young adults could have multimorbidity, which sheds light on the necessity to prevent obesity and other chronic diseases in this vulnerable age group.

On the association between obesity and multimorbidity, our results showed that individuals with obesity and physical multimorbidity exhibit three times higher odds of having suicidal ideation than participants without multimorbidity, and this association was replicable across cycles. This solidifies previous findings (Amiri and Behnezhad, 2018; Xiong et al., 2020) showing physical and/or mental multimorbidity as contributor to the development of suicidal ideation. However, given the root of multimorbidity was not assessed in our study, no direct association between obesity and the different health conditions evaluated can be drawn. While the correlation between obesity and suicidal ideation is understandable, the yet-unknown nature requires additional investigation (Perera et al., 
2016, 2015). In other words, to definitively declare obesity as a cause might be premature. This theory should be probed in future studies.

Further to our findings, the severity of obesity did not have a significant influence on the association with suicidal ideation. While a previous study underlined obesity as a risk factor for suicidal ideation, Dutton et al. (2013) draws this conclusion from the comparison between normal and overweight individuals without considering the disparity between BMI classes as in our study. This leads us to believe that multimorbidity could be the mediating factor of the association between obesity and suicidal ideation. In other words, with multimorbidity, obesity is a risk factor for suicidal ideation regardless of its level.

Regarding the covariates assessed, no distinction between sex and educational status can be confirmed to have a significant effect on the association evaluated, given the inconsistency in the results in our two samples. Nonetheless, it is safe to note that, this disparity can still be notable in other findings (Carpenter et al., 2000). In general, sex disparities within obesity exist on a large scale due to complex sociocultural values (Kanter and Caballero, 2012). For instance, physical appearance holds greater worth in women's eyes than men (Fredrickson and Roberts, 1997; McKinley, 1998) henceforth resulting in poorer self-esteem (Miller and Downey, 1999), perceived weight and depressive mood disorders, all common in people with suicidal behaviours (Mather et al., 2009). For example, in a South Korean study, young women with severe obesity reported the highest rate of suicidal behaviour mainly due to the social stigma and its consequences on women's health (Kim et al., 2016). Thereupon, future studies should focus on unravelling the reality of the matter.

Furthermore, health behaviours, except for tobacco, did not have a significant association amongst the respondents. Our finding is conflicting with a previous study showing that health behaviours have an effect on an individual's mental and physical wellbeing (FARHUD, 2015). For instance, inactivity (Vancampfort et al., 2018) and smoking (Poorolajal and Darvishi, 2016) were positively associated with suicidal ideation. This discrepancy in findings could be the product of our small sample size and the 
measurements assessed in the questionnaires provided, especially regarding PA. Specifically, in our study, only leisure-time PA was used. This classification excludes the fact that PA is a much broader behaviour including several domains (e.g., transportation, occupational, domestic, leisure) and that every movement, even the smallest of ones, counts. More and more studies are showing the impact of small bouts of physical activity on mental health(Bernard et al., 2018). Therefore, through the reduction of PA to leisuretime or guideline requirements, great health benefits are being disregarded (Moore et al., 2012; Warburton and Bredin, 2017). Regarding eating, given it was not assessed in the two cycles, we could not investigate the role of dietary behaviour for young people with obesity and suicidal ideation.

Undoubtedly, obesity is targeting more and more the younger generations. Worsening throughout adulthood, the repercussions can be damaging on physical and mental health (Cheng et al., 2016). Thus, not only is it crucial to grasp the influence of obesity-related comorbidities, but professionals should be aware of the age-specific challenges faced by the youth including suicidal ideation. Overall, our replicated results in a representative sample of the population support evidence underlining the importance of multimorbidity management in the obese population, vulnerable to suicidal ideation. This study shed light on the importance of multimorbidity management, specifically among young adults.

Having said that, several limitations to this study should be acknowledged. For instance, despite the validation of the measurements, the possible bias driven from the self-reported data cannot be omitted. However, the replication of the main association reduced the risk of bias. Moreover, our study included young adults from only one province (Quebec). So, even though the CCHS is assumed to be representative of the Canadian population, we cannot be sure that our results are generalizable to all Canadian youth. Meanwhile, regarding PA, its partial evaluation and inconsistency can be the root of the distinguishable results, as it excludes a great deal of beneficial movements. In addition, the cross-sectional design of the present study is a limitation, as no causality or interference can be concluded. In other words, we lacked the opportunity to investigate the temporal relationship between 
obesity or multimorbidity. To counteract, we tested this association in two independent cycles.

In conclusion, multimorbidity management within obesity-related interventions, could prevent the occurrence of suicidal behaviours amidst Canadian youth. This is particularly intriguing giving suicidality represent a heavy burden among young adults. Future longitudinal studies should untangle the temporal relationship between obesity and multimorbidity in suicidality. Likewise, researchers should look more into the weight of behavioral and individual deviation in this association as their mediator role remains unclear.

\section{Authors Statement}

\section{Contributors}

All authors, Joanne Mattar (JoM), Valérie Chauvin (VC), Jacques Marleau (JaM), Katherina Kavalidou (KK), and Ahmed Jérôme Romain (AJR) have read, revised and approved the final version of the manuscript.

Research Ideation: AJR.

Statistical plan and analyses: AJR, JaM.

Results Interpretations: JoM, AJR, JaM, KK, VC.

Writing of the manuscript: JoM, AJR, KK.

Article Revision: JoM, AJR, KK, VC, JaM.

\section{Role of the funding source}

No financial support was received for the present study.

\section{Conflicts of interest}

No conflict of interest to disclose. 


\section{Ethical considerations}

Ethics approval Participants' approval was assessed by agents from statistics Canada before measurement

\section{References}

Agborsangaya, C.B., Ngwakongnwi, E., Lahtinen, M., Cooke, T., Johnson, J.A., 2013. Multimorbidity prevalence in the general population: the role of obesity in chronic disease clustering. BMC Public Health 13, 1161. https://doi.org/10.1186/1471-245813-1161

Amiri, S., Behnezhad, S., 2018. Body mass index and risk of suicide: A systematic review and meta-analysis. J. Affect. Disord. 238, 615-625. https://doi.org/10.1016/j.jad.2018.05.028

Arensman, E., Scott, V., De Leo, D., Pirkis, J., 2020. Suicide and Suicide Prevention From a Global Perspective. Crisis 41, S3-S7. https://doi.org/10.1027/0227-5910/a000664

Berardelli, I., Corigliano, V., Hawkins, M., Comparelli, A., Erbuto, D., Pompili, M., 2018. Lifestyle Interventions and Prevention of Suicide. Front. Psychiatry 9. https://doi.org/10.3389/fpsyt.2018.00567

Bernard, P., Doré, I., Romain, A.-J., Hains-Monfette, G., Kingsbury, C., Sabiston, C., 2018. Dose response association of objective physical activity with mental health in a representative national sample of adults: A cross-sectional study. PLOS ONE 13, e0204682. https://doi.org/10.1371/journal.pone.0204682

Canada, P.H.A. of, 2020. Suicide in Canada: Key Statistics (infographic) [WWW Document]. URL https://www.canada.ca/en/publichealth/services/publications/healthy-living/suicide-canada-key-statisticsinfographic.html (accessed 6.11.21).

Carpenter, K.M., Hasin, D.S., Allison, D.B., Faith, M.S., 2000. Relationships between obesity and DSM-IV major depressive disorder, suicide ideation, and suicide attempts: results from a general population study. Am. J. Public Health 90, 251-257.

Cheng, H.L., Medlow, S., Steinbeck, K., 2016. The Health Consequences of Obesity in Young Adulthood. Curr. Obes. Rep. 5, 30-37. https://doi.org/10.1007/s13679-0160190-2 
FARHUD, D.D., 2015. Impact of Lifestyle on Health. Iran. J. Public Health 44, 14421444.

Fredrickson, B.L., Roberts, T.-A., 1997. Objectification Theory. Psychol. Women Q. 21, 173-206. https://doi.org/10.1111/j.1471-6402.1997.tb00108.x

Fruh, S.M., 2017. Obesity: Risk factors, complications, and strategies for sustainable long-term weight management. J. Am. Assoc. Nurse Pract. 29, S3-S14. https://doi.org/10.1002/2327-6924.12510

Harris, E.C., Barraclough, B., 1997. Suicide as an outcome for mental disorders. A metaanalysis. Br. J. Psychiatry J. Ment. Sci. 170, 205-228. https://doi.org/10.1192/bjp.170.3.205

Harris, E.C., Barraclough, B.M., 1994. Suicide as an outcome for medical disorders. Medicine (Baltimore) 73, 281-296. https://doi.org/10.1097/00005792-19941100000001

Kanter, R., Caballero, B., 2012. Global gender disparities in obesity: a review. Adv. Nutr. Bethesda Md 3, 491-498. https://doi.org/10.3945/an.112.002063

Kavalidou, K., Smith, D., Der, G., O'Connor, R., 2019. The role of physical and mental multimorbidity in suicidal thoughts and behaviours in a Scottish population cohort study. BMC Psychiatry 19. https://doi.org/10.1186/s12888-019-2032-8

Kavalidou, K., Smith, D., O'Connor, R., 2016. The role of physical and mental health multimorbidity in suicidal ideation. J. Affect. Disord. 209. https://doi.org/10.1016/j.jad.2016.11.026

Kim, D.-K., Song, H.J., Lee, E.-K., Kwon, J.-W., 2016. Effect of sex and age on the association between suicidal behaviour and obesity in Korean adults: a crosssectional nationwide study. BMJ Open 6, e010183. https://doi.org/10.1136/bmjopen2015-010183

Mather, A.A., Cox, B.J., Enns, M.W., Sareen, J., 2009. Associations of obesity with psychiatric disorders and suicidal behaviors in a nationally representative sample. J. Psychosom. Res. 66, 277-285. https://doi.org/10.1016/j.jpsychores.2008.09.008

McKinley, N.M., 1998. Gender Differences in Undergraduates' Body Esteem: The Mediating Effect of Objectified Body Consciousness and Actual/Ideal Weight Discrepancy. Sex Roles 39, 113-123. https://doi.org/10.1023/A:1018834001203

Miller, C.T., Downey, K.T., 1999. A Meta-Analysis of Heavyweight and Self-Esteem. Personal. Soc. Psychol. Rev. 3, 68-84. https://doi.org/10.1207/s15327957pspr0301_4 
Moore, S.C., Patel, A.V., Matthews, C.E., Gonzalez, A.B. de, Park, Y., Katki, H.A., Linet, M.S., Weiderpass, E., Visvanathan, K., Helzlsouer, K.J., Thun, M., Gapstur, S.M., Hartge, P., Lee, I.-M., 2012. Leisure Time Physical Activity of Moderate to Vigorous Intensity and Mortality: A Large Pooled Cohort Analysis. PLOS Med. 9, e1001335. https://doi.org/10.1371/journal.pmed.1001335

Navickas, R., Petric, V.-K., Feigl, A.B., Seychell, M., 2016. Multimorbidity: What do we know? What should we do? J. Comorbidity 6, 4-11. https://doi.org/10.15256/joc.2016.6.72

Pediatric Clinical Advisor, 2007. . Elsevier. https://doi.org/10.1016/B978-0-323-035064.X1000-0

Perera, S., Eisen, R., Bawor, M., Dennis, B., de Souza, R., Thabane, L., Samaan, Z., 2015. Association between body mass index and suicidal behaviors: a systematic review protocol. Syst. Rev. 4, 52. https://doi.org/10.1186/s13643-015-0038-y

Perera, S., Eisen, R.B., Dennis, B.B., Bawor, M., Bhatt, M., Bhatnagar, N., Thabane, L., Souza, R. de, Samaan, Z., 2016. Body Mass Index Is an Important Predictor for Suicide: Results from a Systematic Review and Meta-Analysis. Suicide Life. Threat. Behav. 46, 697-736. https://doi.org/10.1111/sltb.12244

Poorolajal, J., Darvishi, N., 2016. Smoking and Suicide: A Meta-Analysis. PLOS ONE 11, e0156348. https://doi.org/10.1371/journal.pone.0156348

Qin, P., Hawton, K., Mortensen, P.B., Webb, R., 2014. Combined effects of physical illness and comorbid psychiatric disorder on risk of suicide in a national population study. Br. J. Psychiatry J. Ment. Sci. 204, 430-435. https://doi.org/10.1192/bjp.bp.113.128785

Rockett, I.R.H., Wang, S., Lian, Y., Stack, S., 2007. Suicide-associated comorbidity among US males and females: a multiple cause-of-death analysis. Inj. Prev. 13, 311315. https://doi.org/10.1136/ip.2007.015230

Romain, A.J., Marleau, J., Baillot, A., 2018. Impact of obesity and mood disorders on physical comorbidities, psychological well-being, health behaviours and use of health services. J. Affect. Disord. 225, 381-388. https://doi.org/10.1016/j.jad.2017.08.065

Sailer, D., 1998. [Obesity: entrance port to multimorbidity]. Wien. Med. Wochenschr. $1946148,388-392$.

Suicide [WWW Document], n.d. URL https://www.who.int/news-room/factsheets/detail/suicide (accessed 6.11.21). 
Vancampfort, D., Hallgren, M., Firth, J., Rosenbaum, S., Schuch, F.B., Mugisha, J., Probst, M., Van Damme, T., Carvalho, A.F., Stubbs, B., 2018. Physical activity and suicidal ideation: A systematic review and meta-analysis. J. Affect. Disord. 225, 438-448. https://doi.org/10.1016/j.jad.2017.08.070

Vilhjalmsson, R., Kristjansdottir, G., Sveinbjarnardottir, E., 1998. Factors associated with suicide ideation in adults. Soc. Psychiatry Psychiatr. Epidemiol. 33, 97-103. https://doi.org/10.1007/s001270050028

Warburton, D.E.R., Bredin, S.S.D., 2017. Health benefits of physical activity: a systematic review of current systematic reviews. Curr. Opin. Cardiol. 32, 541-556. https://doi.org/10.1097/HCO.0000000000000437

Xiong, F., Wang, L., Shen, L., Guo, W., Li, S., Guan, Q., 2020. The relationship between multimorbidity and suicidal ideation: A meta-analysis. J. Psychosom. Res. 138, 110257. https://doi.org/10.1016/j.jpsychores.2020.110257 